\title{
STEADY STATE FIXED BED COMBUSTION OF WOOD PELLETS. A SIMPLE THEORETICAL MODEL
}

\author{
C. Pinho , \\ T. Ferreira ${ }^{\mathrm{a}}$, \\ E. Marques ${ }^{\mathrm{b}}$, \\ D. Almeida ${ }^{b}$, \\ C. Pereirab, \\ and J. M. Paiva ${ }^{\text {b }}$ \\ ABSTRACT \\ The combustion of biomass follows a sequence of several distinct phases \\ which begin with drying, the subsequent pyrolysis with the release of \\ volatile components that burn in gaseous phase and finally the combustion \\ of carbonaceous resiue, the time consuming step. For small solid fuel \\ boilers, as it is the case of pellet boilers for domestic heating, biomass \\ particles are continuously burned in fixed bed boilers or heaters. In these \\ appliances the furnace is a metallic basket into which fuel particles are \\ thrown and burn. The combustion air is introduced through orifices drilled \\ in this metallic basket although some lateral air entrances are also found. \\ The gaseous flow is a piston flow, the particles fall under gravity on the \\ upper bed surface and as they burn they slowly move downwards until their \\ size is small enough to fall down through the air entrance orifices or are \\ dragged by the upcoming combustion gas flow. So the gaseous current has \\ an up flow movement while the solid particles move downwards. It is \\ assumed that the bed is at uniform temperature and all the average \\ properties of the gaseous flow are constant with the exception of the oxygen \\ concentration that diminishes as the gas flow rises through the bed. The \\ mathematical development of a simple model that allows the calculation of \\ the steady state burning time of a biomass particle in fixed bed, the amount \\ of energy released, the fixed bed size and its particle inventory, is hereby \\ presented. The pedagogical interest of this model is pertinent because it \\ presents, in a synthetic way, the relative importance of the combustion \\ kinetic, the mass transfer mechanism and the reactor fluid dynamics, upon \\ the lifetime of a biochar particle during its combustion process. \\ Received: October 29, 2014 \\ Revised: November 30, 2014 \\ Accepted: December 30, 2014 \\ Keywords: wood combustion, fixed bed, pellets, steady state, reaction \\ control

\section{NOMENCLATURE} \\ $A_{f} \quad$ area of the fixed bed, $\mathrm{m}^{2}$ \\ $C$ molar concentration, $\mathrm{kmol} / \mathrm{m}^{3}$ \\ d diameter, $\mathrm{m}$ \\ $D$ diffusivity, $\mathrm{m}^{2} / \mathrm{s}$ \\ $f_{C} \quad$ carbon to oxygen ratio \\ $H \quad$ bed height, $\mathrm{m}$ \\ $H V_{c}$ heating value of carbon, $\mathrm{J} / \mathrm{kg}$ \\ $i \quad$ reaction index \\ $k_{c} \quad$ reaction rate constant, $\mathrm{m} / \mathrm{s}$ \\ $K_{g l o} \quad$ global combustion constant, $\mathrm{m} / \mathrm{s}$ \\ $L H V_{C 3 H 8}$ lower heating value of propane, $\mathrm{J} / \mathrm{kg}$ \\ $m \quad$ mass, $\mathrm{kg}$ \\ $\dot{m}$ mass flow, $\mathrm{kg} / \mathrm{s}$ \\ $\dot{m}^{\prime \prime} \quad$ mass flux, $\mathrm{kg} /\left(\mathrm{m}^{2} \mathrm{~s}\right)$ \\ $M \quad$ molecular weight, $\mathrm{kg} / \mathrm{kmol}$ \\ $n_{c 1} \quad \mathrm{kmol}$ of carbon, kmol \\ $N \quad$ number of particles \\ $\dot{N}_{p} \quad$ particles rate of supply \\ $Q \quad$ heat released, $\mathrm{J}$ \\ $\dot{Q}$ heat rate, $\mathrm{W}$ \\ $r$ radius, $\mathrm{m}$ \\ Sh Sherwood number \\ $t$ time, $\mathrm{s}$ \\ $U_{f} \quad$ superficial velocity, $\mathrm{m} / \mathrm{s}$ \\ $V \quad$ volume, $\mathrm{m}^{3}$ \\ $Y$ mass fraction

\section{Greek symbols} \\ $\alpha \quad$ parameter defined by Eq. (26) \\ $\beta \quad$ parameter defined by Eq. (27) \\ $\delta \quad$ oxygen concentration reduction factor \\ $\varepsilon_{b} \quad$ bed voidage fraction \\ $\eta \quad$ inter particle competition parameter \\ $\theta \quad$ volatile fraction \\ $\rho$ density, $\mathrm{kg} / \mathrm{m}^{3}$ \\ $\sigma_{c} \quad$ constriction factor \\ $\tau_{b} \quad$ bed tortuosity \\ $\varphi \quad$ burned mass fraction \\ $\phi_{p} \quad$ particle sphericity
}


$\omega \quad$ parameter defined by Eq. (47)

$\chi \quad$ maldistribution bypass factor

\section{Subscripts}

$b \quad$ burned, bed

c carbon

ct carbon total

$c x \quad$ carbon at slice inlet

C carbon

C3H8 propane

eq equivalent

$g$ gas

$i \quad$ initial

ix at slice inlet

oe oxygen inlet

os oxygen outlet

O2 oxygen

$O 2 s$ oxygen surface

$O 2 \infty$ oxygen infinite

$p \quad$ particle

pt particle total

$s$ surface

sf sphere

$\infty \quad$ free stream

\section{INTRODUCTION}

The rising prices and the higher emissions arising from the use of fossil fuels are causing economic and environmental concerns, which are motivating an increase in the use of renewable energies (Verma et al., 2012). The use of biomass as a fuel has important environmental, social and economic advantages that can be exploited and may contribute to sustainable development (Khan et al., 2009). In many countries, the forest is the main source of biomass and represents a significant area at the disposal of the economic activities, to use it as a renewable and valuable energy source. Eucalyptus, oak, and pine are present and dominant species in many Mediterranean areas (ICNF, 2013). Other species, considered invasive, as the genus Cytisus, represent about $4.3 \%$ of the forest area.

In this paper a mathematical model for the steady state fixed bed combustion of wood particles is presented. It is based on a previous model developed by Kanury (1975) but now the importance of the kinetic and of the mass transfer phenomena are simultaneously taken into account.

\section{THE COMBUSTION OF A SINGLE PARTICLE}

After being introduced into the fixed bed the biomass suffers a drying process followed by pyrolysis, with simultaneous combustion of the released volatiles, and finally the solid carbonaceous residue burns. This last stage has a longer duration, controls the total lifetime of the biomass particle and thus requires deeper attention in modeling terms.
To analyze the combustion of batches of carbon particles it is necessary to begin with the combustion of a single spherical particle. According to Kanury (1975), the combustion is assumed in steady state and there is only mass transfer in the radial direction. Making a mass balance of the oxygen transfer towards the burning particle, it can be written that,

$$
D_{O 2}\left[\frac{1}{r^{2}} \frac{\mathrm{d}}{\mathrm{d} r}\left(r^{2} \frac{\mathrm{d} \rho_{O 2}}{\mathrm{~d} r}\right)\right]=V_{r} \frac{\mathrm{d} \rho_{O 2}}{\mathrm{~d} r}
$$

It is assumed that $D_{O 2}$ and $\rho_{O 2}$ are constants, that $\rho_{O 2}=Y_{O 2} \rho_{g}$, where $Y_{O 2}$ is the oxygen mass fraction and that $\rho_{g}$ is the density of the gaseous phase around the burning solid particle. Because it is a steady state process, $\dot{m}_{g}=\rho_{g} v_{r} 4 \pi r^{2}=$ constant $=4 \pi r^{2} \dot{m}^{\prime \prime}$ where $\dot{m}^{\prime \prime}{ }_{g}=\rho_{g} v_{r}$. It is equally true that $r^{2} \dot{m}_{g}{ }^{\prime \prime}=$ constant, and then Eq. (1) becomes

$$
\frac{\mathrm{d}}{\mathrm{d} r}\left(D_{O 2} r^{2} \rho_{g} \frac{\mathrm{d} Y_{O 2}}{\mathrm{~d} r}\right)-\left[\dot{m}^{\prime \prime}{ }_{C} r^{2}\right] \frac{\mathrm{d} Y_{O 2}}{\mathrm{~d} r}=0
$$

where $\dot{m}_{C}{ }_{C}$ is the net mass flux of the gaseous phase around the particle and because of the continuity condition $\left[\dot{m}^{\prime \prime}{ }_{C} r^{2}\right]$ is constant. Integrating Eq. (2) once

$$
D_{O 2} \rho_{g} r^{2} \frac{d Y_{O 2}}{d r}-\left[\dot{m}^{\prime \prime}{ }_{C}{ }^{2}\right] Y_{O 2}=K_{1}
$$

applying this equation for $r=r_{\mathrm{s}}$ and using the boundary condition (Kanury, 1975),

$$
\frac{\dot{m}_{C, s}^{\prime \prime}}{f_{C}}=\left(\left.\rho_{g} D_{O 2} \frac{d Y_{O 2}}{d r}\right|_{s}-\dot{m}_{C, s}{ }^{\prime} Y_{O 2, s}\right)
$$

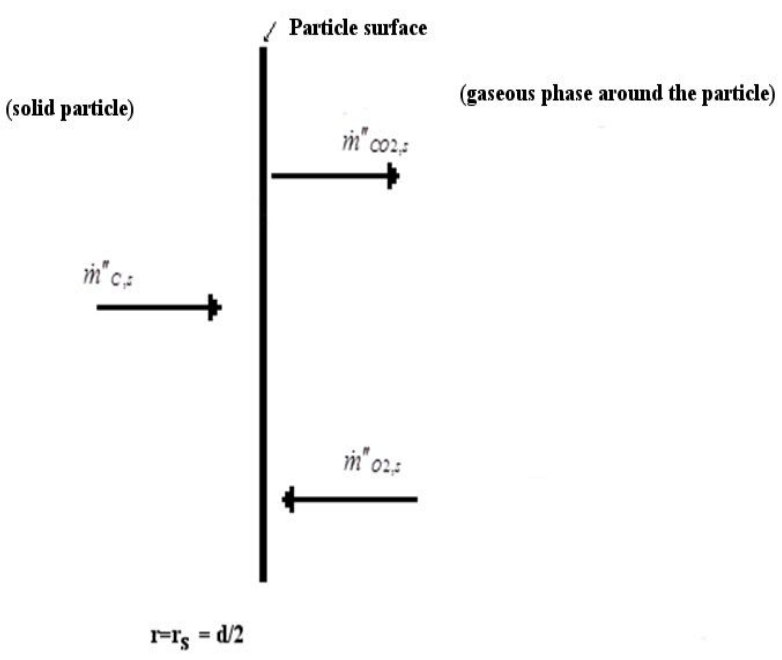

Figure 1. Events taking place at particle surface during combustion. 
reworking the result and integrating with the limits $\left(r=r\right.$ and $\left.Y_{O 2}=Y_{O 2}\right)$ and $(r \rightarrow \infty$ and $\left.Y_{O 2}=Y_{O 2, \infty}\right)$, the following is obtained,

$$
\ln \left(\frac{Y_{O 2}+1 / f_{C}}{Y_{O 2, \infty}+1 / f_{C}}\right)=-\frac{\left[\dot{m}_{C, s}^{\prime \prime} r_{s}^{2}\right]}{\rho_{g} D_{O 2}} \frac{1}{r}
$$

If there is competition between kinetic and mass transfer for the control of the reaction there is a finite oxygen concentration at the surface of the particle, $\mathrm{i}$. e., $Y_{O 2, s} \neq 0$. Then, Eq. (5) becomes,

$$
\ln \left(\frac{Y_{O s, 2}+1 / f_{C}}{Y_{O 2, \infty}+1 / f_{C}}\right)=-\frac{\dot{m}_{C, s}^{\prime \prime} r_{s}^{2}}{\rho_{g} D_{O 2}} \frac{1}{r_{s}}
$$

The carbon consumption rate is a function of the oxygen concentration at its surface,

$$
\dot{m}_{C}=4 \pi r_{s}^{2} M_{C} k_{c} C_{o 2, s}
$$

where $k_{c}$ is the reaction rate constant for the heterogeneous phase $\left(\mathrm{C}+\frac{1}{2} \mathrm{O}_{2} \rightarrow \mathrm{CO}\right)$ reaction. Assuming that the oxygen is a perfect gas, and considering only the mass flux,

$$
\dot{m}_{C, s}=\frac{M_{C}}{M_{O 2}} k_{c} \rho_{g} Y_{O 2, s}
$$

It is now convenient to combine Eqs. (6) and (8) to eliminate $Y_{O 2, s}$. But that is not analytically possible, unless a simplification is carried out. The logarithmic term in Eq. (6) is developed in a Maclaurin series and considering only the first two terms this results in,

$$
\ln \left(\frac{Y_{O 2, s}+1 / f_{C}}{Y_{O 2, \infty}+1 / f_{C}}\right) \approx \ln \left(\frac{1 / f_{C}}{Y_{O 2, \infty}+1 / f_{C}}\right)+f_{C} Y_{O 2, s}
$$

The justification for this approach relies on the fact that $0 \leq Y_{O 2} \leq Y_{O 2, \infty}$ and $0 \leq Y_{O 2, \infty} \leq 0.23$. In other words, $Y_{O 2, s}$ takes small values thus the development in series for $Y_{O 2, s}=0$ will lead to small errors. Combining Eqs. (5), (6) and (9), the following equation is obtained,

$$
Y_{O 2, s}=\frac{\ln \left(f_{C} Y_{O 2, \infty}+1\right)}{\left(f_{C}+\frac{M_{C} k_{c} r_{s}}{M_{O 2} D_{O 2}}\right)}
$$

and because $\dot{m}_{c}=4 \pi r_{s}{ }^{2} \dot{m}_{C, s}$ and doing $\ln \left(f_{C} Y_{O 2, \infty}+1\right) \approx f_{C} Y_{O 2, \infty}$ then,

$$
\dot{m}_{C}=4 \pi r_{s}^{2} \rho_{g} f_{C} Y_{O 2, \infty}\left(\frac{1}{\frac{M_{o 2} f_{C}}{M_{C} k_{c}}+\frac{r_{s}}{D_{o 2}}}\right)
$$

In the above equation $\rho_{g} Y_{O 2, \infty}=\rho_{O 2, \infty}$, $4 \pi r_{s}^{2}=\pi d^{2} \quad$ or $\quad r_{s}=d / 2$ where $d$ is the diameter of the burning particle,

$$
\begin{gathered}
\dot{m}_{C}=\pi d^{2} \rho_{O 2, \infty} \frac{1}{\left(\frac{M_{O 2}}{M_{C} k_{c}}+\frac{d}{2 f_{C} D_{O 2}}\right)} \\
\text { As } \rho_{O 2, \infty}=M_{O 2} C_{O 2, \infty} \text { and } f_{C}=M_{C} / M_{O 2}, \\
\dot{m}_{C}=\pi d^{2} M_{C} K_{g l o} C_{O 2, \infty}
\end{gathered}
$$

and

$$
\frac{1}{K_{g l o}}=\frac{1}{k_{c}}+\frac{d}{2 D_{o 2}}
$$

But, $D_{O 2}$ is the $\mathrm{O}_{2}$ apparent diffusivity in the combustion gases because the burning particle under analysis is a part of a batch of burning particles. Only for the combustion of an isolated particle in pure diffusion conditions $D_{O 2}$ would be the oxygen diffusivity. According to Avedesian and Davidson (1973),

$$
D_{O 2}=\frac{S h D_{g}}{2}
$$

where $D_{g}$ is the effective diffusivity of oxygen in the combustion gases.

In general terms if the model should consider both possibilities, either complete combustion of carbon to $\mathrm{CO}_{2}$ or the incomplete combustion to $\mathrm{CO}$, then the overall combustion resistance would be given by

$$
\frac{1}{K_{g l o}}=\frac{d}{i S h D_{g}}+\frac{1}{k_{c}}
$$

where, $i=1$ for carbon combustion to $\mathrm{CO}_{2}$ and 2 for combustion to $\mathrm{CO}$. 


\section{STEADY STATE COMBUSTION OF SOLID PARTICLES IN A FIXED BED}

For small solid fuel boilers biomass particles are continuously burned in fixed bed. The burner is a metallic basket where fuel particles are let down to be burned in fixed bed mode. The combustion air passes through the holes of this metallic basket. It is assumed that the bed operates as shown in Fig. 2. The gaseous flow is a piston flow, the particles fall under gravity on the upper bed surface and as they burn they slowly move downwards until their size is small enough to fall down through the air entrance orifices or are dragged by the upcoming combustion gas flow. The gaseous current has an up flow movement while the solid particles move downwards. It is considered that the bed is at uniform temperature and all the average properties of the gaseous flow are constant with the exception of the oxygen concentration that reduces as the gas flow rises through the bed.

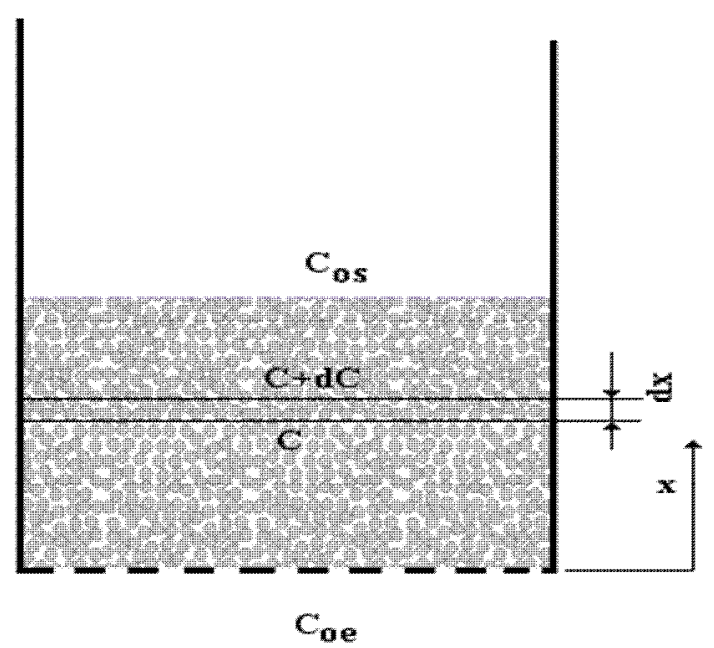

Figure 2. Fixed bed scheme of the combustion of solid particles.

The furnace is formed by an endless number of elemental slices of volume, $\mathrm{d} V$, as depicted in Fig. 3 . Considering one of these elemental slices of volume $\mathrm{d} V$, a well-mixed flow is assumed and the oxygen concentration is uniform inside it and equal to $C_{\infty}$. A particle of diameter $d$ that enters this elemental slice suffers, through combustion, a size reduction that is a function of its combustion rate which is dependent upon its size, the molar concentration of oxygen in the slice and of the combustion mechanism. The elemental time $\mathrm{d} t$ associated to the elemental particle size reduction $\mathrm{d}(d)$ is determined by,

$$
\pi d^{2} K_{g l o} C_{\infty}=-\frac{\mathrm{d}}{\mathrm{d} t}\left(\frac{\rho_{c}}{M_{c}} \frac{\pi d^{3}}{6}\right)
$$

with $K_{g l o}$ defined by Eq. (16). Then,

$$
\mathrm{d} t=-\frac{\rho_{c}}{2 M_{c} \eta C_{\infty}}\left[\frac{d}{i S h D_{g}}+\frac{1}{k_{c}}\right] \mathrm{d}(d)
$$

where $\eta$ is a factor accounting for the inter-particles competition of the available oxygen. But this particle is a part of a set of particles composing the bed that are undergoing a combustion process. Looking into the elemental slice, Fig. 3, there is the descending displacement of the burning particles and the ascending movement of the oxygen. Each kmol of carbon consumes one kmol of oxygen, according to the overall stoichiometry $\mathrm{C}+\mathrm{O}_{2} \rightarrow \mathrm{CO}_{2} \cdot n_{c 1}$ is the number of $\mathrm{kmol}$ of carbon for a single particle of generic diameter $d$,

$$
n_{c 1}=\frac{\rho_{c}}{M_{c}} \frac{\pi d^{3}}{6}
$$

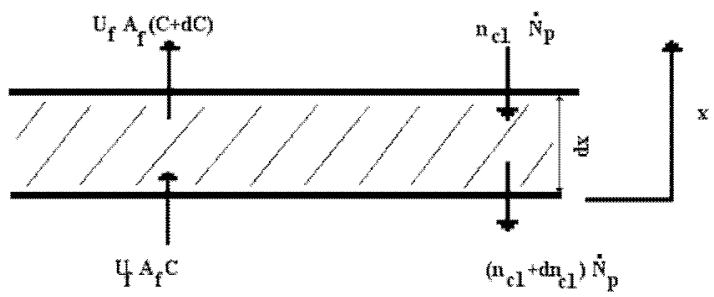

Figure 3. Slice of the fixed bed.

In this slice several particles get in and out per unit of time. Assuming that the particles entering the bed are all of the same size and burn at the same combustion rate, the number of particles crossing a given bed section is a time constant $\dot{N}_{p}$.

$$
\dot{N}_{p}=\frac{6 \dot{m}_{c}}{\rho_{c} \pi d_{i}{ }^{3}}=\frac{6 \dot{m}_{c x}}{\rho_{c} \pi d_{i x}{ }^{3}}
$$

$\dot{N}_{p}$ is the particle rate of supply to the bed while, $\dot{m}_{c}$ is the mass flow rate of carbon at bed inlet, $\dot{m}_{c x}$ is the mass flow rate of carbon at the inlet of the elemental volume $\mathrm{d} V, d_{i}$ is the initial particle diameter at bed inlet and $d_{i x}$ is the particle diameter at the inlet of the elemental volume $\mathrm{d} V$. The oxygen being consumed in the slice of elemental volume $\mathrm{d} V$ is dependent of the carbon being consumed in the same slice,

$$
-U_{f} A_{f} \mathrm{~d} C=\dot{N}_{p} \mathrm{~d} n_{c 1}
$$

but

$$
\mathrm{d} n_{c 1}=\mathrm{d}\left(\frac{\rho_{c} \pi d^{3}}{6 M_{c}}\right)=\frac{\rho_{c} \pi d^{2}}{2 M_{c}} \mathrm{~d}(d)
$$

Combining Eqs. (19), (21) and (22) 


$$
d C=-\frac{3 \dot{m}_{c} d^{2}}{U_{f} A_{f} M_{c} d_{i}^{3}} \mathrm{~d}(d)
$$

There is now a system of two differential equations, Eqs. (18) and (23). Integrating the first one it is possible to know the particle burning time provide that the variation of the oxygen concentration inside the bed, is known. The knowledge of the evolution of the molar concentration of oxygen in the bed, or with the size of the burning particles, is obtained through the integration of Eq. (23) and the result is,

$$
C=\frac{\dot{m}_{c}}{U_{f} A_{f} M_{c}}\left[(1-\varphi)-\frac{d^{3}}{d_{i}{ }^{3}}\right]+C_{o e}
$$

where $\varphi$ stands for the burning fraction of an initial mass flow of particles, $\dot{m}_{c}$, with an initial diameter $d_{i}$. Equation (18) then becomes,

$$
d t=-\frac{\rho_{c} U_{f} A_{f} d_{i}^{3}}{2 \eta \dot{m}_{c} i \operatorname{Sh} D_{g}} \frac{d+\alpha}{\beta-d^{3}} d(d)
$$

where,

$$
\alpha=\frac{i S h D_{g}}{k_{c}}
$$

and

$$
\beta=(1-\varphi) d_{i}{ }^{3}+\frac{U_{f} A_{f} M_{c} C_{o e} d_{i}{ }^{3}}{\dot{m}_{c}}
$$

Equation (25) must now be integrated within the limits,

$$
\begin{array}{cc}
t=0 & d=d_{i} \\
t=t_{\varphi} & d_{\varphi}=(1-\varphi)^{1 / 3} d_{i}
\end{array}
$$

The result for the particle burning time corresponding, to the burned mass fraction $\varphi$, is a rather cumbersome equation,

$$
\begin{aligned}
& t_{\varphi}=\frac{\rho_{c} U_{f} A_{f} d_{i}{ }^{3}}{2 \eta \dot{m}_{c} i \operatorname{Sh} D_{g}} \frac{1}{\beta^{2 / 3}}\left(\frac{\left(\beta^{1 / 3}+\alpha\right) \ln \left(d_{i}{ }^{2}+\beta^{1 / 3} d_{i}+\beta^{2 / 3}\right)}{6}+\right. \\
& \frac{\left(\alpha-\beta^{1 / 3}\right) \arctan \left(\frac{2 d_{i}+\beta^{1 / 3}}{\sqrt{3} \beta^{1 / 3}}\right)}{\sqrt{3}}-\frac{\left(\beta^{1 / 3}+\alpha\right) \ln \left(d_{i}-\beta^{1 / 3}\right)}{3}- \\
& \left.\frac{\left(\beta^{1 / 3}+\alpha\right) \ln \left[(1-\varphi)^{2 / 3} d_{i}{ }^{2}+\beta^{1 / 3}(1-\varphi)^{1 / 3} d_{i}+\beta^{2 / 3}\right]}{6}\right] \\
& \left.-\frac{\left(\alpha-\beta^{1 / 3}\right) \arctan \left[\frac{2(1-\varphi)^{1 / 3} d_{i}+\beta}{\sqrt{3} \beta^{1 / 3}}\right]}{\sqrt{3}}\right\rangle \\
& \left.+\frac{\left(\beta^{1 / 3}+\alpha\right) \ln \left[(1-\varphi)^{1 / 3} d_{i}-\beta^{1 / 3}\right]}{3}\right\rangle
\end{aligned}
$$

However, a simpler approach is to develop the function $\frac{d+\alpha}{\beta-d^{3}}$ in Maclaurin series and to consider only the first two terms, because particle diameters are of mm order, being thus very small. This leads to a simpler equation for the same particle burning time, corresponding to the burned mass fraction $\varphi$,

$$
\begin{aligned}
& t_{\varphi}=\frac{\rho_{c} U_{f} A_{f} d_{i}{ }^{3}}{2 \eta \dot{m}_{c} i \operatorname{Sh} D_{g}} \frac{1}{\beta}\left\{\alpha\left[1-(1-\varphi)^{1 / 3} d_{i}\right]\right. \\
& \left.+\left[1-(1-\varphi)^{2 / 3} \frac{d_{i}{ }^{2}}{2}\right]\right\}
\end{aligned}
$$

Replacing $\alpha$ and $\beta$, Eqs. (26) and (27) respectively, in Eq. (29), leads to,

$$
t_{\varphi}=\frac{\left[1-(1-\varphi)^{1 / 3}\right] d_{i}\left[1-(1-\varphi)^{2 / 3}\right] d_{i}{ }^{2}}{\frac{k_{c}}{2 \eta}\left[\frac{\dot{m}_{c}(1-\varphi)}{\rho_{c}}+M_{c} C_{o e}\right]}
$$

In this last equation, for the steady state burning time of the mass fraction $\varphi$ of a particle $t_{\varphi}$, the first term of the numerator stands for the influence of the kinetics, whilst the second term represents the influence of mass transfer. The denominator exhibits the impact of the reactor fluid mechanics that will, naturally, influence both the kinetic and the mass transfer of the process.

\section{BED PARTICLE INVENTORY AND SIZE}

The solid particles never undergo a complete combustion in the fixed bed because when reaching a small size they may be dragged away in the gaseous flow of the combustion products or may fall through the grate orifices into the ash pit. Thus the subsequent analysis will be based on a given burned mass fraction $\varphi$ and its corresponding burning time $t_{\varphi}$. As already said all particles have the same initial size and burn at the same velocity. The residence time of a given carbon particle, i. e., a biomass particle after pyrolysis, is the corresponding burning time and for a given time instant, the total number of particles in the bed will be,

$$
N_{p t}=t_{\varphi} \dot{N}_{p}
$$

The particle rate of supply $\dot{N}_{p}$ was defined by Eq. (20), $N_{p t}$ is the total number of particles, having a whole range of different sizes, i.e., at different burning stages, that constitute the burning bed. The total mass of solid fuel existing in the fixed bed is a function of the total number of particles in the bed 
$N_{p t}$ and the corresponding average equivalent diameter $d_{e q}$,

$$
m_{c t}=N_{p t} \rho_{c} \frac{\pi d_{e q}{ }^{3}}{6}
$$

These pyrolysed biomass particles are introduced in the bed with an initial diameter $d_{i}$ and burn until reaching a final diameter $(1-\varphi)^{1 / 3} d_{i}$. As the bed operates in a steady state regime, the burning rate of a particle with the equivalent average diameter, multiplied by the number of particles composing the bed, equals the bed feeding rate,

$$
\dot{m}_{c}=\pi d_{e q}{ }^{2} K_{g l_{e q}} M_{c} C_{\infty} N_{p t}
$$

where the global reaction $K_{g l_{e q}}$ constant must be a function of this equivalent diameter

$$
\frac{1}{K_{g l l_{e q}}}=\frac{d_{e q}}{i S h D_{g}}+\frac{1}{k_{c}}
$$

The average molar oxygen concentration in the bed must also be a function of this average equivalent diameter of the burning particles,

$$
C_{\infty}=\frac{\dot{m}_{c}}{U_{f} A_{f} M_{c}}\left[(1-\varphi)-\frac{d_{e q}{ }^{3}}{d_{i}{ }^{3}}\right]+C_{o e}
$$

Replacing Eqs. (20), (31), (34) and (35) in Eq. (33), results in,

$$
\begin{aligned}
& \frac{d_{e q}}{i S h D_{g}}+\frac{1}{k_{c}}=\frac{6 t_{\varphi} M_{c} d_{e q}{ }^{2}}{\rho_{c} d_{i}{ }^{3}} \\
& \left\{\frac{\dot{m}_{c}}{U_{f} A_{f} M_{c}}\left[(1-\varphi)-\frac{d_{e q}{ }^{3}}{d_{i}{ }^{3}}\right]+C_{o e}\right\}
\end{aligned}
$$

which gives the value of $d_{e q}$. The bed height for $H$ a given voidage fraction $\varepsilon_{b}$ will then be

$$
H=\frac{N_{p t} \pi d_{e q}{ }^{3}}{6 \varepsilon_{b} A_{f}}
$$

\section{ENERGY RELEASED DURING THE STEADY STATE COMBUSTION}

For the purpose of the energy balance of the fixed bed furnace, the energy released in the complete combustion of carbon, with a heating value of $H V_{c}$, is given by,

$$
\dot{Q}_{c}=\dot{m}_{c} H V_{c}
$$

But the particles dropped in the fixed bed or furnace are made of wood (biomass) with a $\dot{m}_{b}$ mass flow rate, taking for granted that there is a mass reduction associated with the pyrolysis of biomass particles, if that mass reduction is a fraction $\theta$, then,

$$
\dot{m}_{b}=\theta \dot{m}_{b}+(1-\theta) \dot{m}_{b}
$$

where the carbon mass flow rate fed to the bed is given by

$$
\dot{m}_{c}=(1-\theta) \dot{m}_{b}
$$

and the volatile mass flow, which will be assumed to be composed by $\mathrm{C}_{3} \mathrm{H}_{8}$, as suggested for example by Ribeiro and Pinho (2007), will be given by,

$$
\dot{m}_{v}=\dot{m}_{C 3 H 8}=\theta \dot{m}_{b}
$$

Then, considering the lower heating value of the propane $L H V_{C 3 H 8}$,

$$
\dot{Q}_{v}=\dot{m}_{C 3 H 8} L H V_{C 3 H 8}
$$

the thermal energy released, during $t_{\varphi}$ by the burning of the biomass mass flow $\dot{m}_{b}$, is

$$
Q_{\varphi}=t_{\varphi}\left(\dot{m}_{c} \varphi H V_{C}+\dot{m}_{C 3 H 8} L H V_{C 3 H 8}\right)
$$

\section{THE INTER PARTICLE COMPETITION PARAMETER}

The parameter $\eta$ was defined as the inter particle competition factor for the available oxygen, but it has a wider meaning. When the biomass is sent to the furnace it undergoes a pyrolysis process with the subsequent release of volatile matter. These volatiles in their evolving process will predominantly consume the available oxygen that is being introduced in the furnace air feeding flow. Only the remaining oxygen will be available for the combustion of carbonaceous particles and consequently this reduction of the oxygen availability must be taken into account. On the other end the solid particles constitute a solid bed with close physical contact among them, and their superficial area is not fully available for the combustion reaction. Besides, due to the tortuosity of the particle packing in the fixed bed, the oxygen path towards the particles surface where the reaction takes place will be sinuous. To account for these effects in a simple way a multiplicative factor has to be applied to the oxygen concentration away from the particles.

Following an analogy with mass transfer phenomena in porous particles (Fogler, 2006), this parameter $\eta$ can be defined as, 


$$
\eta=\frac{\varepsilon_{b} \delta}{\tau_{b}}(1-\chi) \sigma_{c}
$$

where $\varepsilon_{b}$ is the bed voidage fraction, $\delta$ is the oxygen concentration reduction factor because of its previous consumption by the released volatiles, $\tau_{b}$ is the bed tortuosity, $\chi$ is a bypass factor to account for flow maldistribution through the fixed bed and $\sigma_{c}$ is a constriction factor. This constriction factor takes into account the fact that particles contact among themselves in the bed reducing the available reaction area (Fogler, 2006). It also accounts in a simple way for the influence of the ashes in the restriction of the oxygen flow towards the surface of the particles.

The biomass after being introduced into the fixed bed suffers a short drying process followed by pyrolysis. In the present analysis it is assumed that the fuel is dry. Assuming that the volatiles released in the pyrolysis are mainly composed by propane, according to the stoichiometry of propane combustion in air $\mathrm{C}_{3} \mathrm{H}_{8}+\frac{(3+8)}{4}\left(\mathrm{O}_{2}+3.76 \mathrm{~N}_{2}\right)$, the corresponding air fuel ratio $(\mathrm{m} / \mathrm{m})$ is,

$$
(A F)_{s t o}=15.66
$$

and remembering that the air has $23 \%(\mathrm{~m} / \mathrm{m})$ of oxygen

$$
\dot{m}_{O_{2}-C_{3} H_{8}}=0.23(A F)_{s t o} \theta \dot{m}_{b}
$$

But the air flow rate supplied to the furnace must be a multiple $\omega$ of the biomass flow rate and thus the total oxygen mass flow will be

$$
\dot{m}_{O_{2}-\text { total }}=0.23 \omega \dot{m}_{b}
$$

In terms of molar flows, Eqs. (47) and (48) give

$$
\dot{n}_{O_{2}-C_{3} H_{8}}=\frac{0.23(A F)_{s t o} \theta \dot{m}_{b}}{M_{O_{2}}}
$$

and

$$
\dot{n}_{O_{2}-\text { total }}=\frac{0.23 \omega \dot{m}_{b}}{M_{O_{2}}}
$$

Finally, the ratio $\delta=\dot{n}_{O_{2}-C} / \dot{n}_{O_{2}-\text { total }}$ is,

$$
\delta=\frac{\omega-(A F)_{e s t} \theta}{\omega}
$$

Table 1 shows some values of $\delta$, as a function of parameters $\omega$ and $\theta$. The adopted values for $\theta$ (0.6 and 0.7 ) correspond to typical mass reductions induced by the pyrolysis process of biomass.

Table 1. Values of $\delta$ function of $\theta$ and $\omega$.

\begin{tabular}{|c|c|c|}
\hline \multirow{2}{*}{$\omega$} & \multicolumn{2}{|c|}{$\theta$} \\
\cline { 2 - 3 } & 0.6 & 0.7 \\
\hline 9.5 & 0.011 & - \\
\hline 9.7 & 0.031 & - \\
\hline 10 & 0.060 & - \\
\hline 10.2 & 0.079 & - \\
\hline 10.5 & 0.105 & - \\
\hline 11 & 0.146 & 0.0073 \\
\hline 11.2 & 0.161 & 0.021 \\
\hline 11.5 & 0.183 & 0.047 \\
\hline 12 & 0.217 & 0.087 \\
\hline
\end{tabular}

It can be seen that the amount of oxygen available for the combustion of the carbon particles in a bed where simultaneously other particles are pyrolysing is rather reduced. For char combustion, where the amount of volatiles being released is negligible, $\delta=1$.

For the calculation of bed tortuosity, the formula of Lanfrey et al. (2010), valid for fixed beds of particles of any shape, can be used,

$$
\tau_{b}=\frac{1.23\left(1-\varepsilon_{b}\right)^{4 / 3}}{\varepsilon_{b} \phi_{p}}
$$

where $\phi_{p}$ is the sphericity of the particles, while the bed voidage fraction can be calculated by the equation of Benhahia and O'Neill (2005),

$$
\varepsilon_{b}=\left(0.1504+\frac{0.2024}{\phi_{p}}\right)+\frac{1.0814}{\left(\frac{d_{b}}{d_{s f}}+0.1226\right)^{2}}
$$

where $d_{s f}$ is the diameter of the sphere of equal volume and $d_{b}$ is the diameter of the fixed bed. This equation is valid for $1.5<d_{b} / d_{s f}<50$ and $0.42<\phi_{p}<1$.

\section{CONCLUSIONS}

The present model was developed based on previously established experimental work on fixed and fluidized bed combustion of biochar particles (Ferreira et al., 2013a, 2013b, 2013c, 2014; Pereira et al., 2013; Pereira and Pinho, 2013, 2014) when it was realized that a simpler theoretical analysis was required for further strengthening the conclusions to 
be obtained from the fixed bed combustion experiments, avoiding or complementing a more demanding CFD analysis. Using the presented theoretical development it is now possible to calculate the burning time of a char particle in fixed bed, to determine the bed size and particle inventory, as well as the energy released during the process.

Work is still being carried out to couple the experimental results with the herein presented approach, both in steady state and in batch combustion testing of biochar particles.

Finally the pedagogical interest of this model must be stressed because it presents, in a synthetic way, the relative importance of the combustion kinetics, the mass transfer and the reactor fluid dynamics, upon the lifetime of a char particle during its combustion process.

\section{ACKNOWLEDGEMENTS}

This work was partially supported by the PTDC/AGR-CFL/114826/2009 grant from the Portuguese Foundation for Science and Technology (FCT). The tests were carried out in the laboratory facilities of ESTV/IPV. The authors wish to express their gratitude to the ESTV board. Tânia Ferreira and Carlos Pinho are thankful to the FEDER funds through the Operational Programme for Competitiveness Factors - COMPETE, ON.2 - O Novo Norte - North Portugal Regional Operational Programme and National Funds through FCT Foundation for Science and Technology under the projects: PEst-C/EQB/UI0511, NORTE-07-0124FEDER-000026 - RL1_ Energy.

\section{REFERENCES}

Avedesian, M. M., and Davidson, J. F., 1973, Combustion of Carbon Particles in a Fluidised Bed, Trans IchemE, Vol. 51, pp.121-131.

Benhaia, F., and O'Neill, K. N., 2005, Enhanced Voidage Correlations for Packed Beds of Various Particle Shapes and Sizes, Particle Science and Technology, Vol. 23, pp. 169-177.

Ferreira, T., Paiva, J. M., and Pinho, C., 2013a, Performance Assessment of a Domestic Boiler using Pellets made from Cytisus scoparius and Cytisus Multiflorus Invasive Shrubs, in: EU BC\&E 2013 $21^{\text {st }}$ European Biomass Conference and Exhibition, (poster 2DV.3.34), Copenhagen, Denmark.

Ferreira, T., Paiva, J. M., and Pinho, C., 2013b, Evaluation of the Performance of a Domestic Pellet Boiler Burning EU Certified and non-certified Commercial PINE pellets, in: $5^{\text {th }}$ International Congress of Energy and Environment Engineering and Management (CIIEM), Lisbon, Portugal.

Ferreira, T., Paiva, J. M., and Pinho, C., 2013c, Performance Assessment of a Domestic Boiler Using Pellets Made from Cytisus multiflorus Invasive Shrubs Compared with Commercial Pine, in: $22^{\text {nd }}$
International Congress of Mechanical Engineering COBEM 2013, Ribeirão Preto, SP, Brazil.

Ferreira, T., Paiva, J. M., and Pinho, C., 2014, Performance Assessment of Invasive Acacia dealbata as a Fuel for a Domestic Pellet Boiler, Chemical Engineering Transactions, Vol. 42, pp.73-78.

Fogler, H. S., 2006, Elements of Chemical Reaction Engineering, Fourth Edition, Pearson Education, Inc.

ICNF, 2013, IFN6 - Áreas dos usos do Solo e das Espécies Florestais de Portugal, Instituto de Conservação da Natureza e das Florestas, Lisbon, Portugal. (in Portuguese)

Kanury, A. M., 1975, Introduction to Combustion Phenomena, Gordon and Breach Science Publishers, New York.

Khan, A., Jong, W., Jansen, P., and Spliethoff, H., 2009, Biomass Combustion in Fluidized Bed Boilers: Potential Problems and Remedies, Fuel Processing Technology, Vol. 90, pp. 21-50.

Lanfrey, P. -Y., Kuzeljevic, Z. V., and Dudukovic, M. P., 2010, Tortuosity Model for Fixed Beds Randomly Packed with Identical Particles, Chemical Engineering Science, Vol. 65, pp. 18911896.

Pereira, C., Marques, E., Paiva, J. M., and Pinho, C., 2013, Cistus ladanifer Shrub - Some Combustion Data from Pelettized Samples: A Small Contribution to Energy Sustainability, in: $22^{\text {nd }}$ International Congress of Mechanical Engineering COBEM 2013, Ribeirão Preto, SP, Brazil.

Pereira, C., and Pinho, C., 2013, Determination of Fluidized Bed Combustion Kinetic and Diffusive Data of Four Wood Chars from the Central Region of Portugal, Energy and Fuels, Vol. 27, pp. 7521-7530.

Pereira, C. C., and Pinho, C., 2014, Influence of Particle Fragmentation and non-sphericity on the Determination of Diffusive and Kinetic Fluidized Bed Biochar Combustion Data, Fuel, Vol. 131, pp. 77-88.

Ribeiro, L., and Pinho, C., 2007, Combustion of Slugs of Propane and Air Moving up Through an Incipiently Fluidized Bed, Combustion Theory and Modelling, Vol. 11, No. 3, pp. 401-425.

Verma, V. K., Bram, S., Delattin, F., Laha, P., Vandendael, I., and Hubin, A., 2012, Agro-pellets for Domestic Heating Boilers: standard Laboratory and Real Life Performance, Applied Energy, Vol. 90, pp. 17-23. 